\title{
Partially Purified Leaf Fractions of Azadirachta indica Inhibit Trypanosome Alternative Oxidase and Exert Antitrypanosomal Effects on Trypanosoma congolense
}

\author{
Abdullah M. Tauheed ${ }^{1}$ (D) Mohammed Mamman ${ }^{1} \cdot$ Abubakar Ahmed $^{2} \cdot$ Mohammed M. Suleiman $^{1}$. \\ Emmanuel O. Balogun ${ }^{3,4}$
}

Received: 10 February 2021 / Accepted: 7 June 2021 / Published online: 22 June 2021

(c) Witold Stefański Institute of Parasitology, Polish Academy of Sciences 2021

\begin{abstract}
Introduction Trypanosomiasis is a neglected disease of humans and livestock caused by single-celled flagellated haemoprotozoan parasites belonging to the genus Trypanosoma.

Purpose Widespread resistance to trypanocidal drugs creates urgent need for new, more effective drugs with potential to inhibit important trypanosome molecular targets.

Methods Nine column chromatographic, partially purified leaf fractions of Azadirachta indica (AIF) were subjected to trypanosome alternative oxidase (TAO) inhibition assay using ubiquinol oxidase assay. The potent TAO inhibitors were evaluated for trypanocidal activities against $T$. congolense in rat model using in vitro, ex vivo, and in vivo assays. Complete cessation or reduction in parasite motility was scored from 0 (no parasite) to 6 (greater than or equal to $6 \times 10^{7}$ trypanosomes/ milliliter of blood), and was used to evaluate the efficacy of in vitro treatments.

Results Only AIF1, AIF2, and AIF5 significantly inhibited TAO. AIF1 and AIF5 produced significant, dose-dependent suppression of parasite motility reaching score zero within $1 \mathrm{~h}$ with $\mathrm{EC}_{50}$ of 0.005 and $0.004 \mu \mathrm{g} / \mu \mathrm{L}$, respectively, while trypanosome-laden blood was still at score six with an $\mathrm{EC}_{50}$ of $44,086 \mu \mathrm{g} / \mu \mathrm{L}$. Mice inoculated with the concentrations at scores 0 and 1 (1-2 moribund parasites) at the end of the experiment did not develop parasitaemia. The two fractions significantly $(p<0.05)$ lowered parasite burden, with the AIF5 exhibiting highest in vivo trypanocidal effects. Packed cell volume was significantly higher in AIF1 $(p<0.05)$ and AIF5 $(p<0.001)$ groups compared to DMSO-treated group. Only AIF5 significantly $(p<0.05)$ lowered malondialdehyde.
\end{abstract}

Conclusion AIF1 and AIF5 offer prospects for the discovery of TAO inhibitor(s).

Keywords Drug discovery $\cdot$ Ex vivo $\cdot$ In vitro $\cdot$ In vivo $\cdot$ Malondialdehyde $\cdot$ Trypanosome metabolism

Abdullah M. Tauheed

mtauheed@abu.edu.ng; amtauheed40@gmail.com

1 Department of Veterinary Pharmacology and Toxicology, Faculty of Veterinary Medicine, Ahmadu Bello University, Zaria, Kaduna, Nigeria

2 Department of Pharmacognosy and Drug Development, Faculty of Pharmaceutical Sciences, Ahmadu Bello University, Zaria, Kaduna, Nigeria

3 Department of Biochemistry, Faculty of Life Sciences, Ahmadu Bello University, Zaria, Kaduna, Nigeria

4 Africa Centre of Excellence for Neglected Tropical Diseases and Forensic Biotechnology (ACENTDFB), Ahmadu Bello University, Zaria, Nigeria

\section{Introduction}

Trypanosomiasis is a disease of humans and livestock caused by several species of single-celled flagellated haemoprotozoan parasites belonging to the genus Trypanosoma. African animal trypanosomiasis (AAT) is caused primarily by infection with Trypanosoma congolense, $T$. vivax, and T. brucei, either singly or in combination [1], while human African trypanosomiasis is caused by $T$. brucei gambiense and $T . b$. rhodesiense. Trypanosomes are transmitted cyclically from infected to uninfected animals through the bite of blood-sucking tsetse flies (Glossina species) [2]. Tsetsetransmitted trypanosomiasis is an important constraint to livestock development in sub-Saharan Africa (SSA) with estimated annual economic losses exceeding US $\$ 4.5$ billion 
[3]. Furthermore, the disease hampers cultivation of over 10 million square kilometers of arable land in SSA.

Widespread resistance to the available antitrypanosomal drugs [4] and weak prospect for vaccine development against trypanosomiasis have rekindled concerted efforts focused on molecular biological target identification and validation as frontier of hope to roll back trypanosomiasis ravaging SSA. Uniquely, trypanosomatids package the first six to seven enzymes of glycolysis into the glycosome, a specialized form of the peroxisome [5]. Glycolysis is especially important for the bloodstream forms of African trypanosomes, which rely exclusively on this pathway for energy production. The compartmentalization of glycolysis in trypanosomatids is accompanied by fundamental differences in allosteric regulation of the pathway compared to most other eukaryotes [6]. Chemical biology has demonstrated distinct inhibition profiles for host and parasite kinases [7], suggesting that selective inhibition of parasite kinases is feasible. Furthermore, both genome-wide and kinome-wide RNAi knockdown screens indicate that several of these enzymes are essential [8] for the parasite survival.

Target-based approach (T-BA) to drug discovery involves screening for inhibitors against a purified protein (e.g., an enzyme) and any compound shown to inhibit the protein or enzyme is optimized for efficacy in a cellular model. T-BA is a good reductionist approach that breaks down diseases to molecular drug targets and facilitates identification of compounds that can inhibit these targets [9]. Trypanosome alternative oxidase (TAO) is an indispensable cytochromeindependent, cyanide-insensitive enzyme in the mitochondrion of bloodstream form of trypanosomes [10] that is responsible for energy production in aerobic condition [11]. Interestingly, this enzyme is absent in mammals which makes it an important target for drug discovery. Although salicylhydroxamic acid (SHAM), ascofuranone, aurachin D, and 3,4-dihydroxybenzoic acid have been shown to inhibit TAO, their in vivo antitrypanosomal effects are far from satisfactory [12]. TAO is also being implicated in other cellular activities, such as, protection against reactive oxygen species and regulation of surface protein expression [12]. The identification and development of new trypanocidal drugs with potential to selectively inhibit important metabolic molecular target in the trypanosomes will revolutionize treatment of trypanosomiasis and will be of immense agricultural and socio-economic benefits to the SSA. Therefore, our aim is to investigate an important ethnomedicinal plant, Azadirachta indica, for potential inhibitor(s) of TAO and demonstrate its antitrypanosomal effects on $T$. congolense using in vitro, ex vivo, and in vivo assays.

Natural products are important sources of compounds for drug discovery. The use of medicinal plants in the management and mitigation of diseases is gaining momentum in different parts of the world, including the developed countries [13, 14]. For example, approximately one quarter of drugs approved for health promotion in the last 2 decades by the Food and Drug Administration and European Medical Agency are from natural products or their derivatives $[15,16]$. Extracts of Azadirachta indica have demonstrated a broad spectrum anti-protozoan activity against $T$. evansi [17], T. brucei rhodesiense [18], T. cruzi [19], Leishmania amazonensis [20], and Toxoplasma gondii [21]. Here, anti-T. congolense activity of hexane extract of $A$. indica is evaluated along with its potential to inhibit TAO.

Azadirachta indica (neem English, dogon yaro/delbejiya Hausa-Nigeria), family Maliaceae is a fast-growing tree of up to $23 \mathrm{~m}$ high with a straight trunk of diameter $4-5 \mathrm{ft}$ and compound, imparipinnate leaves native to India but naturalized and grown widely in tropical and semitropical regions [22]. The ethnopharmacological applications of $A$. inidica include management of tuberculosis, trypanosomiasis, malaria, cancer, diarrhea, gastric ulcer, wound, and fungal infection [22, 23]. Phytochemical constituents such as nimbin, nimbidin, nimbolide, limonoids, $7 \alpha$-acetyl-15 $\beta$ methoxy-29 methylene 7,15-deoxo nimbolide, 2-oxo-3-deacetyl salannin, and $7 \alpha$-hydroxy- $15 \beta$-hydroxy-7,15-deoxo nimbin have been isolated from the plant $[21,22]$.

\section{Materials and Methods}

\section{Plant Collection and Identification}

Leaves of Azadirachta indica were collected in area A, behind Botanical Garden of the Department of Botany on the main campus of Ahmadu Bello University ( $A B U$ ), Zaria, Nigeria. A sample of the plant, comprising leaves and seeds, was sent to the Herbarium Unit, Department of Botany, Faculty of Life Science, ABU, Zaria for identification and the voucher number 900151 was given for reference. The leaf was dried in the laboratory to a constant weight and pulverized with mortar and pestle for extraction.

\section{Plant Extraction, Concentration, and Purification of the Fractions}

The solvents and thin-layer chromatography (TLC) plates used in the study were products of Merck KGaA, Germany. Two kg of the pulverized leaf was successively and exhaustively extracted with hexane, ethyl acetate, and methanol with Soxhlet apparatus. Based on data from in vitro preliminary study, hexane extract was the most potent extract and it was subjected to column chromatography (liquid chromatography), whereupon 128 fractions were collected. Monitoring of these fractions on TLC using their $\mathrm{R}_{\mathrm{f}}$ values yielded nine fractions termed partially purified fractions (AIF1-F9). In each case, concentration to dryness was done with rotary 
evaporator at $60{ }^{\circ} \mathrm{C}$, and the extracts and fractions were weighed and kept in appropriately labeled bottles in fridge $\left(4{ }^{\circ} \mathrm{C}\right)$ before usage.

\section{Experimental Animals}

The rats and mice used in the study were from outbred stock, obtained from the Animal House of the Department of Veterinary Pharmacology and Toxicology, ABU, Zaria. The animals were acclimatized for 2 weeks in the laboratory before the commencement of the experiment. They were kept in clean plastic cages with wood shavings as beddings. The bedding was changed twice weekly. The rats were fed on standard rat feed and given access to clean water ad libitum. Approval for the use of the animals was given by the Ethical Committee on Animal Use and Care, Ahmadu Bello University, Zaria, with reference number: ABUCAUC/2019/005, in line with international best practices.

\section{Test Organism}

Trypanosoma congolense was obtained from the Nigerian Institute for Trypanosomiasis Research, Kaduna, Kaduna State, Nigeria. Continuous passage was used to maintain the parasite in the laboratory during the course of the experiment. Each cycle of passage was done when parasitaemia of a donor rat was in the range of $8.4 \times 10^{7}$ parasites per milliliter of blood (64 parasites per field). Briefly, $3 \mathrm{ml}$ of blood was obtained from a donor rat by cardiac puncture into a $5 \mathrm{ml}$ syringe and emptied into a vial containing $9 \mathrm{ml}$ of normal saline (NS). Approximately $1 \times 10^{6}$ parasites in $0.2 \mathrm{ml}$ blood/NS solution was injected, intraperitoneally, into a rat previously unexposed to trypanosomal infection.

\section{Enzyme Inhibition Assay}

The recombinant trypanosome alternative oxidase (rTAO) used in this study was kindly provided by Dr. Emmanuel O. Balogun, Department of Biomedical Chemistry, Graduate School of Medicine, University of Tokyo, Japan. Inhibitory effect of the PPFs and crude methanol extract of Azadirachta indica was carried out on TAO using the ubiquinol-1 oxidase assay [24]. Briefly, $10 \mu \mathrm{g}$ of each fraction/extract (sample) was pre-incubated with $75 \mathrm{ng}$ rTAO and $50 \mathrm{mM}$ Tris- $\mathrm{HCl}, \mathrm{pH} 7.4$ for $2 \mathrm{~min}$ in a $1 \mathrm{~mL}$ quartz cuvette. Thereafter, $150 \mu \mathrm{M}$ of ubiquinol-1 was added to initiate the reaction. A pre-incubated rTAO, Tris- $\mathrm{HCl}$ and ubiquinol- 1 only served as control. The inhibitory activity of the samples on the enzyme was measured by recording change in absorbance at $278 \mathrm{~nm}$ with UV-Vis spectrophotometer (Cary 300 UV-Vis Agilent Technologies).

\section{Determination of Median Lethal Dose of the Extract and PPFs $\left(\mathrm{LD}_{50}\right)$}

The limit dose of $5000 \mathrm{mg} / \mathrm{kg}$ [25] was used to determine the $\mathrm{LD}_{50}$ of extracts and fractions of $A$. indica. Healthy young adult non-pregnant nulliparous female rats with average weight of $130 \mathrm{~g}$ were used. Feed was withheld from $7.00 \mathrm{pm}$ before the administration at 8.00 am and for additional $4 \mathrm{~h}$ after the administration. One rat was dosed daily with $5000 \mathrm{mg} / \mathrm{kg}$ and observed for a period of $48 \mathrm{~h}$. A total of five rats were dosed and observed daily for any sign of toxicity for 14 days.

\section{Experimental Design}

\section{In vitro and Ex vivo Studies}

For the in vitro and ex vivo assays, $50 \mu \mathrm{L}$, of $0.1,0.2,1,2$, 10 , and $20 \mu \mathrm{g} / \mu \mathrm{L}$ of PPFs, crude extract of $A$. indica and diminazene aceturate (DA, positive control) was pipetted into individual wells of 96-well round-bottom microtiter plate. Fifty microliters of trypanosome-laden blood, T-LB $\left(8.6 \times 10^{7}\right.$ parasite $/ \mathrm{mL}$ of blood $)$ was added to each well containing the different concentrations of PPFs, extract, and DA $(7 \mathrm{mg} / \mathrm{mL}$, B. No AT00161A, Alivira Animal Health Limited, India), giving a final concentration of 0.05, 0.1, $0.5,1,5$, and $10 \mu \mathrm{g} / \mu \mathrm{L}$, respectively. Each experiment was conducted in triplicate. Similarly, three wells each with $50 \mu \mathrm{L}$ of $\mathrm{T}-\mathrm{LB}+50 \mu \mathrm{L}$ of $5 \%$ dimethyl sulfoxide (DMSO) and $50 \mu \mathrm{L}$ of T-LB only served as negative and untreated controls, respectively. The set-up was incubated at $25{ }^{\circ} \mathrm{C}$, while the efficacy of treatment measured as complete immobilization or reduction of parasite motility relative to negative control was observed at $1 \mathrm{~h}$ interval for $6 \mathrm{~h}$ and scored [26] (Table 1). At the end of the $6 \mathrm{~h}$ in vitro study, ex vivo inoculation of mice was done. Accordingly, concentration that produced complete cessation of parasites motility was immediately inoculated into mouse and observed daily

Table 1 Scoring chart for assessing trypanosome viability under in vitro study [26]

\begin{tabular}{lcl}
\hline $\begin{array}{l}\text { Score } \\
\text { fomes per microscopic } \\
\text { field }\end{array}$ & $\begin{array}{l}\text { Number of trypano- } \\
\text { Inference }\end{array}$ \\
\hline 0 & 0 & $\begin{array}{l}\text { No viable (motile) parasite in } \geq 20 \\
\text { fields } \\
\text { Moribund parasites in } \geq 20 \text { fields }\end{array}$ \\
1 & $1-2$ & Motile parasites in $\geq 3$ fields \\
2 & $3-5$ & Motile parasites in $\geq 3$ fields \\
3 & $6-10$ & Motile parasites in $\geq 3$ fields \\
4 & $11-20$ & Motile parasites in $\geq 3$ fields \\
5 & $21-40$ & Motile parasites in $\geq 3$ fields \\
6 & $>40$ &
\end{tabular}


for possible development of parasitaemia. Similarly, the concentrations that did not immobilize the parasite at the end of the experiment were also individually inoculated into one mouse each and observed daily for possible development of parasitaemia.

\section{In vivo Study}

Twenty-five adult male rats weighing $130-170 \mathrm{~g}$ were divided into five groups of five rats each. The rats were infected intraperitoneally with $10^{6} \mathrm{~T}$. congolense in $0.2 \mathrm{~mL}$ of PBS-diluted blood. Upon establishment of parasitaemia, rats in groups I, II, and III were treated with crude methanol extract, AIF1 and AIF5 of A. indica at $200 \mathrm{mg} / \mathrm{kg}$, respectively, rats in groups IV and $\mathrm{V}$ were treated with diminazene aceturate, DA ( $7 \mathrm{mg} / \mathrm{kg})$, and 5\% dimethyl sulfoxide $(2 \mathrm{~mL} / \mathrm{kg})$, which served as positive and negative controls, respectively. Five additional rats that were neither infected nor treated in group VI served as normal control group. All the treatments were administered via the intraperitoneal route and for 5 days except DA, which was given once. The efficacy of the treatments was assessed daily using a drop of blood collected from rat tails onto a microscope slide, covered with cover slip, and observed under a light microscope at $\times 400$ magnification. Degree of elimination/suppression of parasite multiplication from/in the systemic circulation of the experimental animals, hematological parameters, and serum malondialdehyde (MDA) were used to evaluate in vivo antitrypanosomal effects of the treatments. The rats were observed for three weeks, beginning from the day of commencement of treatment. Thereafter, the experiment was terminated by jugular venesection, and blood and serum were collected for the evaluation of hematological parameters and MDA, respectively.

\section{Hematological Profile}

One ml of blood from each rat was collected into EDTA bottle and analyzed for packed cell volume (PCV), hemoglobin concentration, and total and differential leukocyte and platelet counts. Also, erythrocytic indices mean corpuscular volume, mean corpuscular hemoglobin, and mean corpuscular hemoglobin concentrations were determined using autoanalyzer (Mindray Hematology Analyzer BC3600, Hoevelaken, Netherlands).

\section{Determination of Serum MDA}

Three $\mathrm{ml}$ of blood sample from each rat was equally collected into plain bottle without anticoagulant and was allowed to clot, and centrifuged at $1000 \mathrm{~g}$ for $15 \mathrm{~min}$ to obtain the serum. Serum MDA was determined as described by Draper and Hadley [27]. Briefly, $0.5 \mathrm{~mL}$ of serum sample was mixed with $2.5 \mathrm{~mL}$ of $100 \mathrm{~g} / \mathrm{L}$ trichloroacetic acid (TCA) solution in a centrifuge tube and placed in boiling water bath for $15 \mathrm{~min}$. After cooling it under running tap water, it was centrifuged at $1000 \mathrm{~g}$ for $10 \mathrm{~min}$. Thereafter, $2 \mathrm{~mL}$ of the supernatant was taken and added to $1 \mathrm{ml}$ of $6.7 \mathrm{~g} / \mathrm{L}$ thiobarbituric acid (TBA) in a test tube and returned to the bath again for another $15 \mathrm{~min}$. It was cooled again under running tap water and its absorbance was taken at $532 \mathrm{~nm}$ with spectrophotometer (Spectrum lab 23A China). A tube with $1 \mathrm{~mL}$ of $10 \%$ TCA and $1 \mathrm{ml}$ of $0.67 \%$ TBA served as blank. The intensity of pink coloration indicates the extent of lipid peroxidation.

\section{Data Analysis}

Results obtained were expressed as mean \pm standard error of mean and subjected to one-way analysis of variance followed by Tukey post-test. The results of the in vitro studies were subjected to Kruskal-Wallis and Dunn's post-tests. GraphPad prism version 5.0 was used and values of $p<0.05$ were considered statistically significant. Excel was used to calculate $\mathrm{EC}_{50}$ using the linear regression equation of best fit $y=a x+b$; where $y=$ dependent variable $\left(\mathrm{EC}_{50}\right), x=$ explanatory variable, $a=$ intercept (the value of $\mathrm{y}$ when $x=0$ ), and $b=$ slope.

\section{Results}

\section{Enzyme Inhibition Assay}

Out of the nine partially purified fractions of $A$. indica that were subjected to TAO inhibition assay, only fractions one and five (AIF1 and AIF5) significantly inhibited the enzyme compared to the negative control. AIF4 also inhibited the enzyme, though not statistically significant $(p<0.05)$, when compared to the control group. Crude methanol extract of $A$. indica (AICM) significantly inhibited TAO compared to the negative control. Nonetheless, the inhibitory effect of AICM was not comparable to the three fractions (Fig. 1).

\section{In vitro and Ex vivo Studies}

The effect of in vitro trypanocidal study of PPFs is expressed in percentage inhibition of parasite motility and shown in Fig. 2. AIF1, AIF5, and AICM demonstrated significant and dose-dependent suppression of parasite motility in the in vitro study. The fractions achieved score zero within $60 \mathrm{~min}$ at the highest concentration of $10 \mu \mathrm{g} / \mu \mathrm{L}$, while DA and T-LB were still at score six. AICM, however, reached score zero within 120 min. Paradoxically, AICM produced complete cessation of motility of the parasites at as low as $1 \mu \mathrm{g} / \mu \mathrm{L}$ with an effective concentration $\left(\mathrm{EC}_{50}\right)$ of $0.0033 \mu \mathrm{g} /$ 


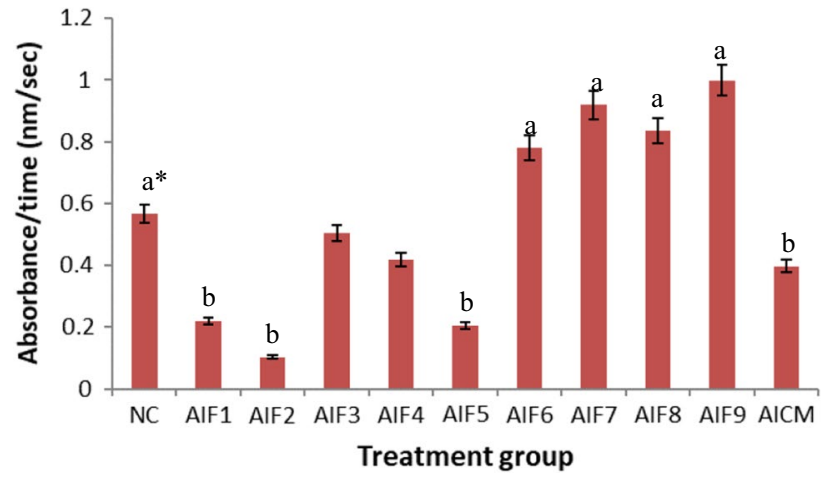

Fig. 1 Enzyme inhibition assay of partially purified fractions and extract of $A$. indica on TAO. Bars with different letters show significant inhibition $(p<0.05)$ of the enzyme. $\mathbf{a}^{*}$ means significantly lowered $(p<0.05)$ than a. NC normal control, AI Azadirachta indica, $F$ partially purified fraction (PPF), $C M$ crude methanol

$\mu \mathrm{L}$, lower (greater effect) than AIF1 and AIF5 which produced similar effect at $5 \mu \mathrm{g} / \mu \mathrm{L}$ with the $\mathrm{EC}_{50}$ of 0.005 and $0.004 \mu \mathrm{g} / \mu \mathrm{L}$, respectively. However, DA and T-LB produced an $\mathrm{EC}_{50}$ of 0.068 and $44,086 \mu \mathrm{g} / \mu \mathrm{L}$, respectively. Furthermore, ALCM exhibited significant reduction of parasite motility up to $0.1 \mu \mathrm{g} / \mu \mathrm{L}$ when compared to 0.5 and $1 \mu \mathrm{g} /$ $\mu \mathrm{L}$ of AIF1 and AIF5, respectively. The values of $\mathrm{EC}_{50}$ are shown in Table 2

The concentrations of the fractions and AICM that completely immobilized the parasite motility and immediately inoculated into the mice did not develop parasitaemia within 4 weeks. Similarly, with the exception of AICM at $0.1 \mu \mathrm{g} /$ $\mu \mathrm{L}$, all the concentrations that reached score one at the end
Table 2 Concentrations of the half-maximal response of the experimental groups

\begin{tabular}{lllllr}
\hline Group & AICM & AIF1 & AIF5 & DA & T-LB \\
\hline $\mathrm{EC}_{50}(\mu \mathrm{g} / \mu \mathrm{L})$ & 0.003 & 0.0045 & 0.0041 & 0.0675 & 44.086 \\
$R^{2}$ & 0.9624 & 0.955 & 0.974 & 0.971 & 0.930 \\
\hline
\end{tabular}

AI Azadirachta indica, $C M$ crude methanol, $F$ Fraction (PPF), DA diminazene aceturate, $T-L B$ trypanosome-laden blood, $E C_{50}$ minimum effective concentration

of the experiment and inoculated into mice did not develop parasitaemia after 1 month post-inoculation (Table 3 ). The highest concentration of DA $(10 \mu \mathrm{g} / \mu \mathrm{L})$ was at score 4 , while DMSO was at score 6 at the point of inoculation into the mice.

\section{In vivo Studies of PPFs}

AICM significantly reduced levels of parasitaemia on days 5 and $7(p<0.01)$ and day $6(p<0.05)$ compared to the DMSO group (Fig. 3). Days 8-14 showed marked significant $(p<0.001)$ reduction in the levels of parasitaemia compared to DMSO-treated group. Furthermore, AIF1 produced significant $(p<0.05)$ decrease in the level of parasitaemia from systemic circulation on day 4 compared to the DMSOtreated group. Days 5-14 and 16 recorded marked significant $(p<0.001)$ reduction in the levels of parasitaemia compared with the DMSO group. The levels of significant reduction in parasitaemia were recorded on day $4(p<0.05)$ and days $5-16(p<0.001)$. DA completely eliminated the parasites
Fig. 2 Percentage inhibition of $T$. congolense by partially purified fractions in the in vitro study. AI Azadirachta indica, $F$ partially purified fraction, $C M$ crude methanol, $D A$ diminazene aceturate, DMSO (5\%) dimethyl sulfoxide, $T-L B$ Trypanosomeladen blood

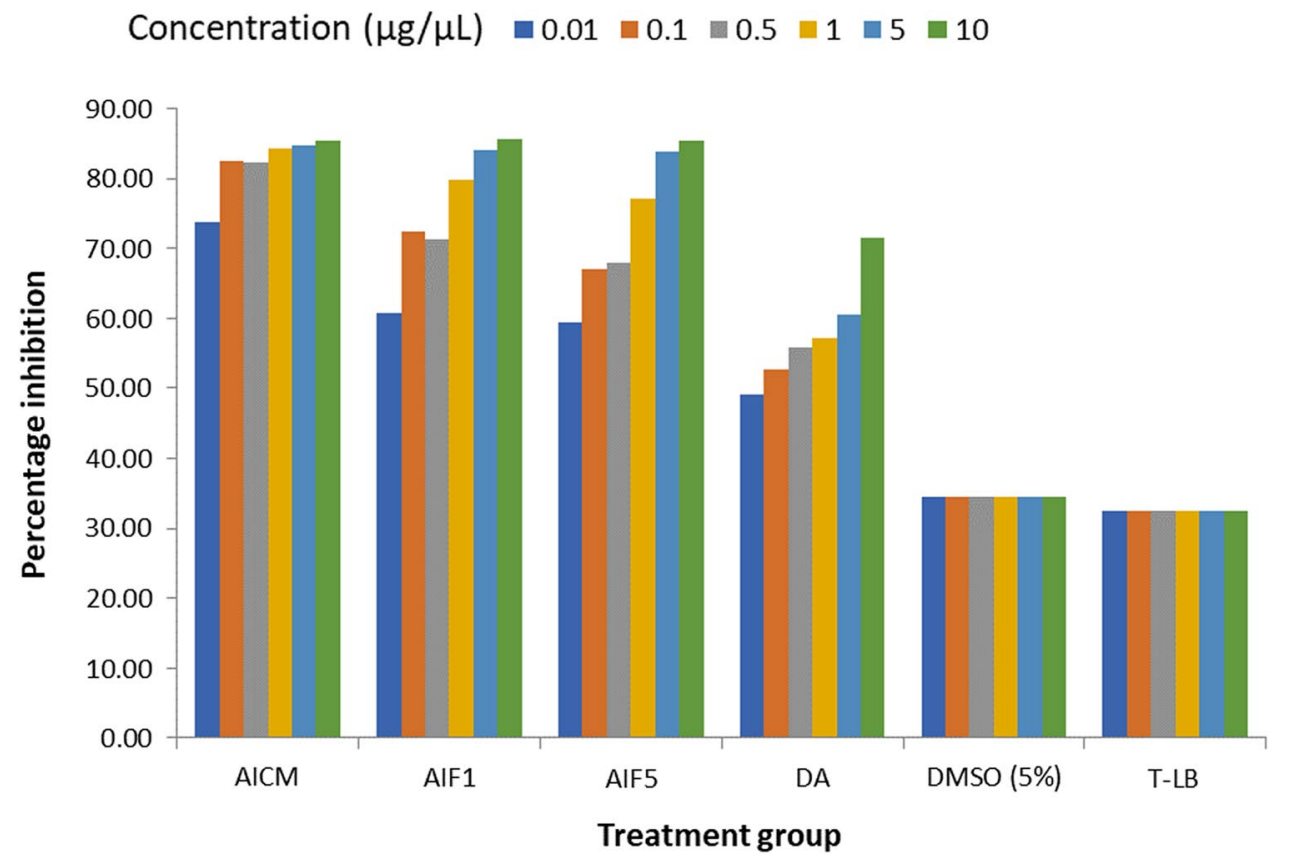


Table 3 Ex vivo antitrypanosomal assessment of the partially purified fractions

\begin{tabular}{llllll}
\hline $\begin{array}{l}\text { Concentra- } \\
\text { tions }(\mu \mathrm{g} / \mu \mathrm{L})\end{array}$ & $\begin{array}{l}\text { AICM } \\
x / y\end{array}$ & AIF1 & AIF5 & DA & DMSO 5\% \\
\hline 0.05 & $0 / 3$ & $0 / 3$ & $0 / 3$ & $0 / 3$ & $0 / 3$ \\
0.1 & $1 / 3^{\mathrm{b}}$ & $0 / 3$ & $0 / 3$ & $0 / 3$ & $0 / 3$ \\
0.5 & $3 / 3^{\mathrm{a}, \mathrm{b}}$ & $3 / 3^{\mathrm{a}, \mathrm{b}}$ & $0 / 3$ & $0 / 3$ & $0 / 3$ \\
1 & $3 / 3$ & $3 / 3^{\mathrm{a}, \mathrm{b}}$ & $3 / 3^{\mathrm{a}, \mathrm{b}}$ & $0 / 3$ & $0 / 3$ \\
5 & $3 / 3$ & $3 / 3$ & $3 / 3$ & $0 / 3$ & $0 / 3$ \\
10 & $3 / 3$ & $3 / 3$ & $3 / 3$ & $3 / 3$ & $0 / 3$ \\
\hline
\end{tabular}

$x / y$ survived mice/inoculated mice

AI Azadirachta indica, $F$ partially purified fraction, $C M$ crude methanol, $D A$ diminazene aceturate, $D M S O$ dimethyl sulfoxide

${ }^{\text {a }}$ One or two of the wells completely immobilized the trypanosomes

${ }^{\mathrm{b}}$ Contained 1-2 sluggish parasites per microscopic field at the point of inoculation into the mice

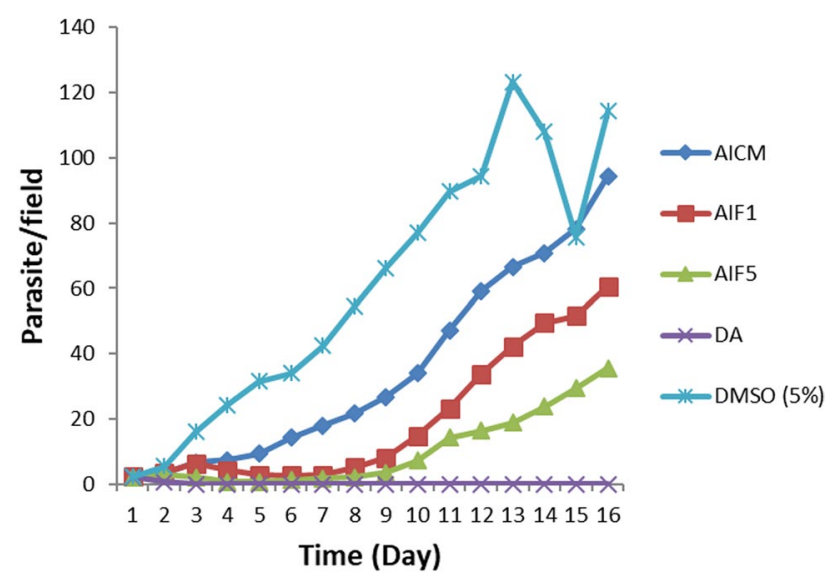

Fig. 3 In vivo result of the partially purified fractions on mean daily levels of parasitaemia. AI Azadirachta indica, $F$ partially purified fraction, $C M$ crude methanol, $D A$ diminazene aceturate, $D M S O$ dimethyl sulfoxide

from systemic circulation of the treated rats within $48 \mathrm{~h}$ of the treatment and without relapse parasitaemia.

The overall performance of each treatment group on reduction of multiplication of $T$. congolense in the systemic circulation of the experimental rats is shown in Fig. 4. The two fractions and crude extract significantly suppressed multiplication of $T$. congolense compared to DMSO. AIF5 exhibited highest in vivo suppressive effect on parasitaemia compared to AIF1 and AICM-treated groups.

\section{Hematology}

The AIF5-treated rats had significantly $(p<0.001)$ higher PCV than the 5\% DMSO-treated rats. Similarly, the PCV of rats in AIF1 was significantly $(p<0.05)$ higher than

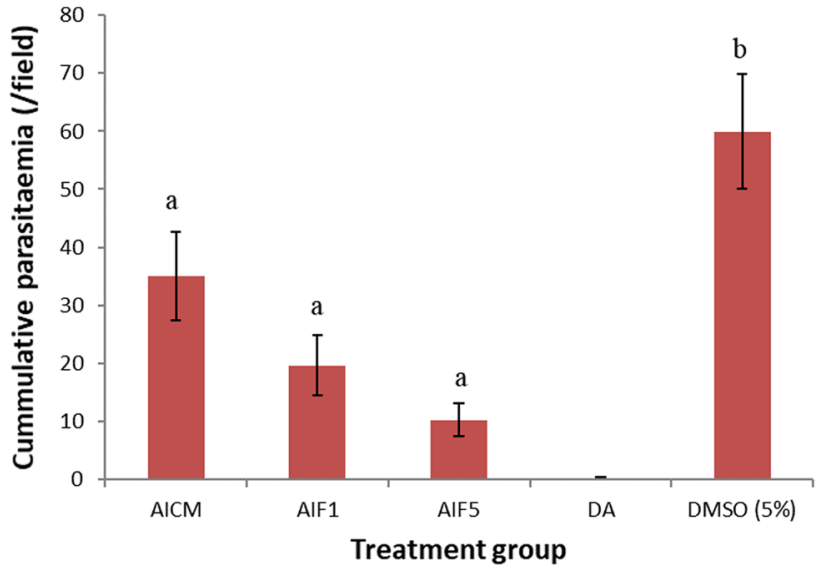

Fig. 4 Cumulative mean parasite reduction as a measure of overall performance of each treatment group. Bars with different letters indicate significant $(p<0.05)$ suppression of parasite multiplication. $A I$ Azadirachta indica, $F$ partially purified fraction, $C M$ crude methanol, $D A$ diminazene aceturate, $D M S O$ dimethyl sulfoxide

the negative control group (Table 4). The PCV of AICM $(33.10 \pm 1.38)$ was non-statistically $(p>0.05)$ higher than the DMSO-treated group $(26.96 \pm 2.19)$. DA-treated rats maintained significantly $(p<0.001)$ higher PCV than the DMSO group. The extract and the fraction-treated rats showed higher mean corpuscular hemoglobin when compared with DMSO group, though not statistically significant $(p>0.05)$. AICM produced significantly $(p<0.01)$ higher white blood cell count and lymphocytes compared to DMSO.

\section{Serum Malondialdehyde}

Effect of treatment with partially purified fractions of $A$. indica on serum malondialdehyde (MDA) is shown in Fig. 5. AICM and AIF5 significantly decreased serum MDA at $p<0.05$ and $p<0.001$, respectively, when compared to the DMSO-treated group. Furthermore, AICM and AIF5 showed significantly $(p<0.05)$ lowering effect on serum MDA compared to AIF1. Although serum MDA of AIF1 is lower than that of DMSO, it is not statistically significant $(p>0.05)$. Interestingly, AIF5 exhibited non-significantly $(p>0.05)$ lower serum MDA compared to the DA. Finally, DA significantly $(p<0.05)$ lower serum MDA compared to DMSO.

\section{Discussion}

A primary step in target-based drug discovery is the identification of specific putative molecular target(s) that play leading role in the pathophysiology of the disease [28]. Trypanosome alternative oxidase (TAO) is an important enzyme for energy requirements and survival of trypanosomes and has 
Table 4 Hematologic parameters of experimental T. congolense-infected rats treated with partially purified fractions and crude extract of Azadirachta indica

\begin{tabular}{|c|c|c|c|c|c|c|}
\hline Parameter & AICM & AIF1 & AIF5 & DA & DMSO (5\%) & $\mathrm{NC}$ \\
\hline $\mathrm{RBC} 10^{6} / \mu \mathrm{L}$ & $4.90 \pm 0.16$ & $5.05 \pm 0.22$ & $4.66 \pm 0.39$ & $7.14 \pm 0.46$ & $4.92 \pm 0.17$ & $8.26 \pm 0.42$ \\
\hline HGB g/dL & $9.38 \pm 0.58$ & $9.66 \pm 0.34$ & $8.48 \pm 0.78$ & $13.78 \pm 0.61$ & $8.54 \pm 0.40$ & $14.47 \pm 0.72$ \\
\hline PCV\% & $33.10 \pm 1.38$ & $36.48 \pm 1.80^{\mathrm{a}}$ & $41.20 \pm 2.31^{\mathrm{a}^{* *}}$ & $43.86 \pm 2.88^{\mathrm{a}^{* *}}$ & $26.96 \pm 2.19^{\mathrm{b}}$ & $49.50 \pm 1.01^{\mathrm{a}^{* * *}}$ \\
\hline MCV fL & $63.06 \pm 2.55$ & $58.02 \pm 2.10$ & $58.86 \pm 2.23$ & $53.66 \pm 1.20$ & $58.86 \pm 1.43$ & $56.60 \pm 1.04$ \\
\hline $\mathrm{MCH}$ pg & $19.26 \pm 0.78$ & $19.18 \pm 0.38$ & $19.02 \pm 0.49$ & $18.48 \pm 0.80$ & $17.82 \pm 0.29$ & $17.87 \pm 0.60$ \\
\hline $\mathrm{MCHC}$ g/dL & $28.72 \pm 0.53$ & $29.42 \pm 1.24$ & $30.00 \pm 0.60$ & $31.24 \pm 1,21$ & $29.80 \pm 0.25$ & $31.43 \pm 1.67$ \\
\hline WBC $10^{3} / \mu \mathrm{L}$ & $56.70 \pm 11.78^{\mathrm{a}^{\mathrm{a*}}}$ & $12.44 \pm 1.12$ & $9.50 \pm 1.12$ & $10.54 \pm 0.88$ & $15.14 \pm 3.82^{b}$ & $11.13 \pm 1.37$ \\
\hline
\end{tabular}

Different superscripts show significant different, ${ }^{*} 0.05,{ }^{* *} 0.001$

AI Azadirachta indica, $F$ partially purified fraction, $C M$ crude methanol, $D A$ diminazene aceturate, $D M S O$ dimethyl sulfoxide, $N C$ normal control

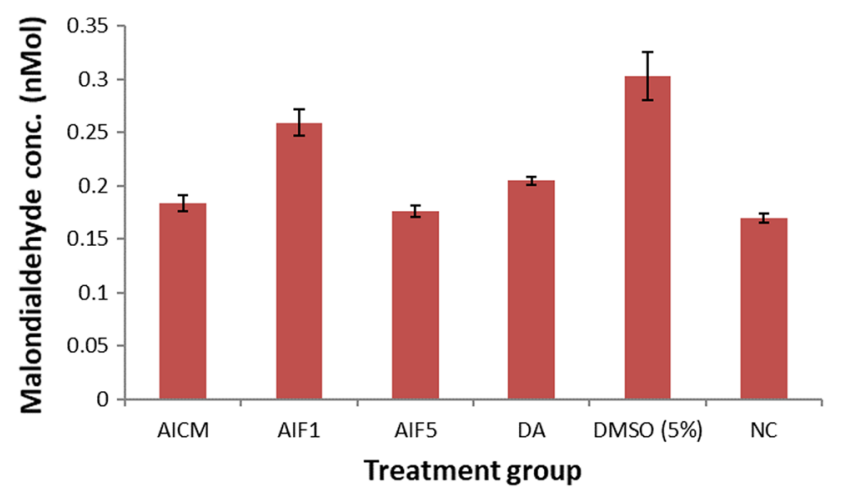

Fig. 5 Serum malondialdehyde of T. congolense-infected rats treated with PPFs. Bars with different letters indicate significant different ${ }^{*} p<0.05,{ }^{*} p<0.0001$. AI Azadirachta indica, $F$ partially purified fraction, $C M$ crude methanol, $D A$ diminazene aceturate, $D M S O$ dimethyl sulfoxide, $N C$ normal control

been the focus of new drug discovery against trypanosomiasis [12]. Our enzyme-inhibition assay of TAO shows that out of the nine partially purified fractions of $A$. indica tested, only AIF1, AIF2, and AIF5 significantly inhibited TAO. The inhibition of TAO by these fractions coupled with significant in vitro and in vivo activities reported in the present study will rekindle hope in the search for new metabolic inhibitors of trypanosome. This is particularly important as resistance to the only defined target inhibitor of ornithine decarboxylase, eflornithine has been reported [29]. Our results show that the AIF1 and AIF5 contain bioactive metabolites capable of potently inhibiting energy metabolism of bloodstream form of trypanosome via inhibition of TAO.

The AIF1 and AIF5 rapidly produced complete cessation of $T$. congolense motility within $1-3 \mathrm{~h}$ of incubation. This effect is superior to long incubation time required for diamidines and amidines to exhibit in vitro antitrypanosomal effect [30]. Of these fractions, fraction five of Azadirachta indica designated as AIF5 had the lowest $\mathrm{EC}_{50}$ and, therefore, exhibited highest in vitro antitrypanosomal effect. The absence of parasite from systemic circulation of the inoculated mice following complete cessation of motility further supports the findings of the in vitro study. Kaminsky et al. [31] had earlier reported the use of drug incubation infectivity test (DIIT) following in vitro enumeration of cultured trypanosomes. Therefore, we observed that DIIT gives more objectivity to the enumeration of cultured trypanosomes and offers alternative to other more expensive methods of screening compounds for in vitro antitrypanosomal activity. These methods include an indicator of metabolic cell function Alamar Blue assay [32], fluorometric measurement of hydrolysed BCECF [33], cleavage of the tetrazolium salt MTT (3,(4,5-dimethylethiazol-2-yl)-2,5-diphenyltetrazolium bromide) [34], colorimetric measurement of $\mathrm{pH}$ change due to pyruvate production [35], and incorporation of radioactive nucleotides, $\left[{ }^{3} \mathrm{H}\right]$ hypoxanthine [36]. These assays are expensive, laborious, and time-consuming, and in addition, the use of radioactive nucleotides poses a serious health hazard. Furthermore, these tests are not suitable for routine field screening in Africa where simple, inexpensive assays are needed [32]. Taken together, the $6 \mathrm{~h}$ DIIT described here could be of immense value in resource-constrained settings like SSA and field screening where simple, sensitive and cheap techniques, and rapid results are required to identify potential drug candidates. It can also hasten compound profiling for antitrypanosomal study in the face of high-throughput screening.

The ability of the partially purified fractions to significantly suppress proliferation of $T$. congolense in the systemic circulation of the treated rats demonstrates promising in vivo antitrypanosomal effects of $A$. indica. Anaemia is one of the most detrimental consequences of trypanosomiasis and most consistent clinicopathological findings [37]. More commonly, a sharp decrease in PCV of livestock suspected of trypanosomiasis is often used to aid diagnosis of African animal trypanosomiasis. Anaemia often persists after 
the first wave of parasitaemia even when parasitaemia has declined to lowest level in systemic circulation $[38,39]$. The significantly lower PCV in the present study lends credence to this. The generally upheld mechanisms of trypanosomeinduced anaemia include immune-mediated galectin-3 erythrophagocytosis and macrophage migration inhibitory factor-mediated suppression of erythropoiesis [40], destruction of the erythrocyte membrane from either the oxidative damage [39, 41], infection-induced anti-variable surface glycoprotein-mediated haemolysis [42], or cleavage of sialic acid of the erythrocyte membrane by trypanosome sialidase [43] and haemodilution [44]. Treatment with extract and fractions of $A$. indica was able to alleviate $T$. congolensemediated anaemia in the present study and maintained significantly high PCV. Thus, the plant, particularly AIF5 could be of immense value in enhancing livestock productivity in trypanosome-endemic areas owing to its ability to maintain significantly adequate PCV. Naessens [45] earlier observed that control of anaemia is more beneficial to the survival and productivity of trypanosome-ridden animals than the control of parasitaemia. Mean corpuscular volume and mean corpuscular hemoglobin concentration are erythrocytic indices used to assess the nature and extent of anaemia. Our findings show that these parameters were not significantly different from the values of the negative control, which translates into normocytic-normochromic anaemia. This is in agreement with the findings of earlier researchers who reported normocytic-normochromic anaemia in rodents [46], cats [47], and dog [48] infected with trypanosome. Normocytic-normochromic anaemia is the type of anaemia found in chronic inflammatory diseases [49]. Indeed, we observed decrease in the inflammatory cells in the fraction-treated groups. It follows that $T$. congolense-mediated anaemia is not the result of down-regulation or suppression of myeloid tissue. The high WBC and lymphocyte counts in the infected rats in our study agree with the finding of [46]. However, treatment with fractions of A. indica kept leukocytosis at basal levels. This may not be unconnected with promising antitrypanosomal effects that keep parasitaemia at bay and thus prevent activation of inflammatory processes.

The ability of AIF5 to significantly lower serum levels of MDA in the treated rats implies amelioration of lipid peroxidation of cell membrane, especially erythrocytes and thus reduction in serum MDA. Maintenance of integrity of cell membrane is a function of its lipid content. Conditions such as trypanosomiasis that produce high free radicals beyond the capacity of natural antioxidant system predispose to peroxidation of lipid membrane with resultant higher MDA in the serum $[50,26]$. The negative group produced significantly highest MDA, indicating excessive peroxidation of lipid membrane and perturbation of the T. congolense-infected rats. MDA is the most important metabolite of omega- 6 polyunsaturated fatty acid and commonly used biomarker of oxidative stress [51]. Furthermore, MDA triggers inflammation and aggravates onset of clinical disease [50]. Hence, AIF5 protects cell membrane against deleterious effect of MDA mediated by lipid peroxidation and maintains fluidity of cell membrane. The superior in vivo antitrypanosomal effects of AIF5 observed in the present study could be explained by its ability to significantly increase $\mathrm{PCV}$ and decrease MDA.

\section{Conclusion}

We conclude that AIF1 and AIF5 potently inhibited TAO and demonstrated significant antitrypanosomal effects. Furthermore, the significantly higher in vivo antitrypanosomal effects of AIF5 can be attributed to its greater ability to increase packed cell volume and lower malondialdehyde, in addition to inhibition of TAO. Optimization of AIF5 may open another opportunity for the synthesis of a novel trypanosome metabolic inhibitor.

Acknowledgements We are grateful to Mr. Bashir Ibrahim and Dr. Na'imatu A. Sani for assistance in the TAO inhibition assay; and column fractionation and in vitro assay, respectively. TAO was graciously donated by E.O. Balogun. We thank Messrs. Dennis Otie and Abdul Wahab Hashim for technical assistance, and Sanusi Musa who catered for the animals.

Author contributions AMT: conceptualization, methodology, resources, formal analysis, investigation, and writing - original draft; MM: methodology, validation, supervision, and writing-review and editing; AA: methodology, validation, and supervision; MMS: methodology; EOB: conceptualization, methodology, resources, validation, supervision, and writing — review and editing.

Funding No funding was received to assist with the preparation of this manuscript.

\section{Declarations}

Conflicts of interest The authors declare that they have no competing interests.

Ethical approval Permission for the use of rats was given by the Ethical Committee on Animal Use and Care, Ahmadu Bello University, Zaria (reference number: ABUCAUC/2019/005).

Consent to participant Not applicable.

\section{References}

1. Steverding D (2008) The history of African trypanosomiasis. Paras Vect. https://doi.org/10.1186/1756-3305-1-3

2. Diall O, Cecchi G, Wanda G, Argilés-Herrero R, Vreysen MJB, Cattoli G et al (2017) Developing a progressive control pathway for African animal trypanosomosis. Trends Parasitol 33:499-509 
3. Yaro M, Munyard KA, Stear MJ, Groth DM (2016) Combatting African animal trypanosomiasis (AAT) in livestock: the potential role of trypanotolerance. Vet Parasitol 225:43-52

4. Giordani F, Morrison LJ, Rowan TG, de Koning H, Barrett MP (2016) The animal trypanosomiases and their chemotherapy: a review. Parasitol 143:1862-1889

5. Haanstra JR, van Tuijl A, Kessler P, Reijnders W, Michels PAM, Westerhoff HV (2008) Compartmentation prevents a lethal turboexplosion of glycolysis in trypanosomes. Proc Nat Acad Sci 105:17718-17723

6. Brimacombe KR, Walsh MJ, Liu L, Vásquez-Valdivieso MG, Morgan HP, McNae I (2014) Identification of ML251, a potent inhibitor of T. brucei and T. cruzi phosphofructokinase. ACS Med Chem Letters 5:12-17. https://doi.org/10.1021/ml400259d

7. Urbaniak MD, Mathieson T, Bantscheff M, Eberhard D, Grimaldi R, Miranda-Saavedra D (2012) Chemical proteomic analysis reveals the drugability of the kinome of Trypanosoma brucei. ACS Chem Biol 7:1858-1865. https://doi.org/10.1021/cb300326z

8. Jones NG, Thomas EB, Brown E, Dickens NJ, Hammarton TC, Mottram JC (2014) Regulators of Trypanosoma brucei cell cycle progression and differentiation identified using a kinome-wide RNAi screen. PLoS Path. https://doi.org/10.1371/journal.ppat. 1003886

9. Harrison RK (2016) Phase II and phase III failures 2013 2015. Nat Rev Drug Discov 15:817-818. https://doi.org/10.1038/nrd.2016. 184

10. Chaudhuri M, Ott RD, Hill GC (2006) Trypanosome alternative oxidase: from molecule to function. Trends Parasitol 22:484-491

11. Gualdron-Lopez M, Brennand A, Avilan L, Michels PA (2013) Translocation of solutes and proteins across the glycosomal membrane of trypanosomes, possibilities and limitations for targeting with trypanocidal drugs. Parasitol 140:1-20

12. Menzies SK, Tulloch LB, Florence GJ, Smith TK (2018) The trypanosome alternative oxidase: a potential drug target? Parasitol 145:175-183. https://doi.org/10.1017/S0031182016002109

13. Thomford NE, Senthebane DA, Rowe A, Munro D, Seele P, Id AM (2018) Natural products for drug discovery in the 21st century: innovations for novel drug discovery. Int J Mol Sci 19:1578 1607. https://doi.org/10.3390/ijms19061578

14. Yatoo MI, Dimri U, Gopalakrishnan A, Karthik K, Gopi M, Khandia R (2017) Beneficial health applications and medicinal values of pedicularis plants: a review. Biomed Pharmacother 95:1301-1313

15. Newman DJ, Cragg GM (2016) Natural products as sources of new drugs from 1981 to 2014. J Nat Prod 79:629-661. https://doi. org/10.1021/acs.jnatprod.5b01055

16. Patridge E, Gareiss P, Kinch MS, Hoyer D (2016) An analysis of FDA-approved drugs: natural products and their derivatives. Drug Discov Today 21:204-207

17. Fahrimal Y, Maghfirah S, Rinidar R, Azhar A, Asmilia N, Erina E (2017) Anti-trypanosoma activity of ethnolic extract of neem leaf (Azadirachta indica) on Trypanosoma evansi in rats (Rattus norvegicus). Indo J Vet Sci. https://doi.org/10.21157/j.ked.hewan. v11i1.5450

18. Githua M, Hassanali A, Keriko J, Murilla G, Ndungu M, Nyagah G (2010) New antitrypanosomal tetranotriterpenoids from Azadirachta indica. Afr J Trad CAM 7:207-213

19. Yanes A, Finol HJ, Hasegawa M (2004) Effects of Azadirachta indica and Melia azedarach (Meliaceae) extracts from leaves on Trypanosoma cruzi growth and ultrastructure. J Submicrosc Cytol Pathol 36:149-154

20. Carneiro S, Carvalho FAA, Santana LCLR, Sousa APL, Neto JMM, Chaves MH (2012) The cytotoxic and antileishmanial activity of extracts and factions of leaves and fruits of Azadirachta indica (A Juss.). Biol Res 42(2):111-116. https://doi.org/10.4067/ S0716-97602012000200002
21. Melo EJT, Vilela KJ, Carvalho CS (2011) Effects of aqueous leaf extracts of Azadirachta indica A.Juss. (neem) and Melia azedarach L. (Santa Barbara or cinnamon) on the intracellular development of Toxoplasma gondii. Rev Bras Plantas med 13(2):215-222

22. Alzohairy MA (2016) Therapeutics role of Azadirachta indica (neem) and their active constituents in diseases prevention and treatment. Evidence-Based Comp Alter Med. https://doi.org/10. 1155/2016/7382506

23. Tiwari R, Verma AK, Chakraborty S, Dhama K, Singh SV (2014) Neem (Azadirachta indica) and its potential for safeguarding health of animals and humans: a review. J Biol Sci 14:110-123. https://doi.org/10.3923/jbs.2014.110.123

24. Kido Y, Sakamoto K, Nakamora K, Hadara M, Suzuki T, Yabu Y (2010) Purification and kinetic characterization of recombinant alternative oxidase from Trypanosoma brucei brucei. Biochim Biophysic Acta 1797:443-450

25. Organization for Economic Co-operation and Development (2008) OECD guidelines for the testing of chemicals. OECD/OCDE 425:1-27

26. Tauheed AM, Mamman M, Ahmed A, Suleiman MM, Balogun EO (2020) In vitro and in vivo antitrypanosomal efficacy of combination therapy of Anogeissus leiocarpus Khaya senegalensis and potash. J Ethnopharmacol 258:112805. https://doi.org/10.1016/j. jep.2020.112805

27. Draper HH, Hadley M (1990) Malondialdehyde determination as index of lipid peroxidation. Met Enzymol 186:421-431

28. Moffat JG, Vincent F, Lee JA, Eder J (2017) Opportunities and challenges in phenotypic drug discovery: an industry perspective. Nat Rev Drug Discov 16:531-543. https://doi.org/10.1038/nrd. 2017.111

29. Barrett M, Croft SL (2012) Management of trypanosomiasis and leishmaniasis. Brit Med Bull 104:175-196. https://doi.org/10. 1093/bmb/lds031

30. Gillingwater K, Kunz C, Braghiroli C, Boykin DW, Tidwell RR, Brun $\mathrm{R}$ (2017) In vitro, ex vivo, and in vivo activities of diamidines against Trypanosoma vivax. Antimicrob Agents Chemoth. https://doi.org/10.1128/AAC.02356-16

31. Kaminsky R, Gumm ID, Zweygarth E, Chuma F (1990) A drug incubation infectivity test (DIIT) for assessing resistance in trypanosomes. Vet Parasitol 34:335-343. https://doi.org/10.1016/03044017(90)90079-Q

32. Räz B, Iten M, Grether-Bühler Y, Kaminsky R, Brun R (1997) The Alamar Blue assay to determine drug sensitivity of African trypanosomes $\mathrm{Tb}$ rhodesiense and $\mathrm{Tb}$ gambiense in vitro. Acta Tropic 68:139-147. https://doi.org/10.1016/s0001-706x(97)00079-x

33. Obexer W, Schmid C, Brun R (1995) A novel in vitro screening assay for trypanocidal activity using the fluorescent dye BCECFAM. Trop Med Parasitol 46:45-48

34. Ellis JA, Fish WR, Sileghem M, McOdimba F (1993) A colorimetric assay for trypanosome viability and metabolic function. Vet Parasitol 50:143-149. https://doi.org/10.1016/0304-4017(93) 90015-F

35. Zinsstag J, Brun R, Gessler M (1991) A new photometric assay for testing trypanocidal activity in vitro. Parasitol Res 77:33-38. https://doi.org/10.1007/BF00934381

36. Brun R, Kunz C (1989) In vitro drug sensitivity test for Trypanosoma brucei subgroup bloodstream trypomastigotes. Acta Trop 46:361-368. https://doi.org/10.1016/0001-706X(89)90048-X

37. Balogun EO, Balogun JB, Yusuf S, Inuwa HM, Ndams IS, Sheridan P (2014) Anemia amelioration by lactose infusion during trypanosomosis could be associated with erythrocytes membrane de-galactosylation. Vet Parasitol 199:259-263

38. Ouma J, Mwangi J, Mdachi R, Njiru N, Ndung U (2001) Validation of indirect Elisa systems for the serodiagnosis of bovine Trypanosomosis in endemic areas of Kenya. FAO/IAEA report. 
In: Animal Trypanosomosis: diagnosis and epidemiology. Backhuys Publishers, The Netherlands, pp 93-98

39. Noyes HA, Alimohammadian MH, BrassFuchsGailus-Durner AMAHV (2009) Mechanisms controlling anemia in trypanosoma congolense infected mice. PLoS ONE 4:e5170. https://doi.org/10. 1371/journal.pone.0005170

40. Stijlemans B, De Baetselier P, Magez S, Van Ginderachter JA, De Trez C (2018) African trypanosomiasis-associated anemia: the contribution of the interplay between parasites and the mononuclear phagocyte system. Front Immunol 9:218. https://doi.org/ 10.3389/fimmu.2018.00218

41. Tauheed AM, Shittu SH, Suleiman MM, Habibu B, Kawu MU, Kobo PI (2016) In vivo ameliorative effects of methanol leaf extract of Lawsonia inermis Linn on experimental Trypanosoma congolense infection in Wistar rats. Int J Vet Sci Med 4:33-40. https://doi.org/10.1016/j.ijvsm.2016.10.005

42. Rifkin MR, Landsberger FR (1990) Trypanosome variant surface glycoprotein transfer to target membranes: a model for the pathogenesis of trypanosomiasis. Proc Nat Acad Sci 89:801-805. https://doi.org/10.1073/pnas.87.2.801

43. Saad SB, Ibrahim MA, Jatau ID, Shuaibu MN (2019) Trypanostatic activity of geranylacetone: mitigation of Trypanosoma congolense-associated pathological pertubations and insight into the mechanism of anemia amelioration using in vitro and in silico models. Exp Parasitol 201:49-56

44. Tilg H, Ulmer H, Kaser A, Weiss G (2002) Role of IL 10 for induction of anemia during inflammation. J Immunol 169:2204 2209. https://doi.org/10.4049/jimmunol.169.4.2204

45. Naessens J (2006) Bovine trypanotolerance: a natural ability to prevent severe anemia and hemophagocytic syndrome? Int J Parasitol 36:521-528
46. Do Carmo GM, Baldissera MD, Vaucher RA, Rech VC, Oliveira CB, Sagrillo MR et al (2015) Effect of the treatment with Achyrocline satureioides (free and nanocapsules essential oil) and diminazene aceturate on hematological and biochemical parameters in rats infected by Trypanosoma evansi. Experim Parasitol 149:39-46. https://doi.org/10.1016/j.exppara.2014.12.005

47. Da Silva AS, Wolkmer P, Costa MM, Lopes ST, Monteiro SG (2011) Anemia in cats infected by Trypanosoma evansi. Comp Clin Pathol 11:393-396. https://doi.org/10.1007/ s00580-010-1009-2

48. Franciscato C, Lopes STA, Teixeira MMG, Monteiro SG, Wolkmer P, Garmatz BC (2007) Dog naturally infected by Trypanosoma evansi in Santa Maria, RS. Brasil Ciência Rural 37:288-291. https://doi.org/10.1590/S0103-84782007000100049

49. Sarma PR (1990) Red cell indices. In: Walker HK, Hall WD, Hurst JW (eds) Clinical methods: The history, physical, and laboratory examinations, 3rd edn. Butterworths, Boston

50. Lee JC-Y, Durand T (2019) Lipid peroxidation: analysis and applications in biological systems. Antioxid 40:1-2. https://doi.org/10. 3390/antiox 8020040

51. Ayala A, Munoz MF, Arguelles S (2014) Lipid peroxidation: production, metabolism, and signaling mechanisms of malondialdehyde and 4-hydroxy-2-nonenal. Oxid Med Cell Longev 360438:1-31. https://doi.org/10.1155/2014/360438

Publisher's Note Springer Nature remains neutral with regard to jurisdictional claims in published maps and institutional affiliations. 